\title{
Brief Behavioural Activation treatment for depressed adolescents delivered by non- specialist clinicians: a case illustration
}

Article

Accepted Version

Creative Commons: Attribution-Noncommercial-No Derivative Works 4.0

Pass, L., Hodgson, E., Whitney, H. and Reynolds, S. (2018) Brief Behavioural Activation treatment for depressed adolescents delivered by non-specialist clinicians: a case illustration. Cognitive and Behavioral Practice, 25 (2). pp. 208224. ISSN 1077-7229 doi:

https://doi.org/10.1016/j.cbpra.2017.05.003 Available at https://centaur.reading.ac.uk/71973/

It is advisable to refer to the publisher's version if you intend to cite from the work. See Guidance on citing.

Published version at: http://www.sciencedirect.com/science/article/pii/S1077722917300536

To link to this article DOI: http://dx.doi.org/10.1016/j.cbpra.2017.05.003

Publisher: Elsevier

All outputs in CentAUR are protected by Intellectual Property Rights law, including copyright law. Copyright and IPR is retained by the creators or other copyright holders. Terms and conditions for use of this material are defined in the End User Agreement. 


\section{CentAUR}

Central Archive at the University of Reading

Reading's research outputs online 
Title: Brief Behavioural Activation treatment for depressed adolescents (Brief BA) by nonspecialist clinicians: A case illustration

\section{ACCEPTED MANUSCRIPT}

Note: This manuscript has been accepted for publication in Cognitive and Behavioral Practice, and should be referenced as:

Pass, L., Hodgson, E., Whitney, H., \& Reynolds, S. (2017). Brief Behavioral Activation

Treatment for Depressed Adolescents Delivered by Nonspecialist Clinicians: A Case

Illustration. Cognitive and Behavioral Practice. DOI: 10.1016/j.cbpra.2017.05.003

The published article can be accessed here:

https://www.journals.elsevier.com/cognitive-and-behavioral-practice/

(C) 2017. This manuscript version is made available under the CC-BY-NC-ND 4.0

license http://creativecommons.org/licenses/by-nc-nd/4.0/ 


\begin{abstract}
Behavioural Activation (BA) can be as effective as Cognitive Behaviour Therapy (CBT) for the treatment of depression in adults, but to date, there is little research with adolescents. This is problematic given the recognised need to increase access to evidence based interventions for depression in young people. We have developed a new adaptation of Brief Behavioural Activation (Lejuez, Hopko, Acierno, Daughters, \& Pagoto, 2011) specifically for young people; Brief Behavioural Activation for depressed adolescents (Brief BA). In this paper we use a case example with session by session measurement to show how a nonspecialist clinician can deliver this intervention successfully. We discuss the key themes arising from this training case, challenges the clinician faced, and how these were managed through training and supervision.
\end{abstract}

Keywords: Behavioral Activation, BA, adolescents, supervision, training 
Brief Behavioural Activation treatment for depressed adolescents (Brief BA) by nonspecialist clinicians: A case illustration

Depression in young people is a common and debilitating psychological disorder that is associated with many negative long term impacts, including poor academic attainment (Verboom, Sijtsema, Verhulst, Penninx, \& Ormel, 2014), lower employment prospects and wages (Johar \& Truong, 2014), mental health difficulties in adulthood (Fergusson, Boden, \& Horwood, 2007) and suicide (Cash \& Bridge, 2009). Prevalence rates vary but in the United States it is estimated that before they reach 19 years old, around $11 \%$ of young people will experience clinically significant depression (Avenevoli, Swendsen, He, Burstein, \& Merikangas, 2015). Worldwide, around $2.6 \%$ of young people experience depression at any one time (Polanczyk, Salum, Sugaya, Caye, \& Rohde, 2015). Depression in adolescence is characterized by low mood or irritability, and/or loss of interest and pleasure in previously enjoyed activities (along with other cognitive and biological symptoms including cognitive difficulties, sleep/appetite disturbance, feelings of worthlessness and psychomotor retardation/agitation).

\section{CBT for Adolescent Depression}

Cognitive Behavioural Therapy (CBT) is an evidence based treatment for depression in adolescents and is recommended both in the United Kingdom (National Institute for Health \& Care Excellence; NICE, 2015) and United States (Birmaher et al., 2007). Unfortunately access to CBT is often limited by availability of qualified psychological therapists. Training routes and accreditation schemes vary between countries but registered CBT therapists are typically qualified at a Masters or Doctoral level (BABCP, 2012). Even when there are clear economic arguments to support increasing the number of qualified CBT therapists (Layard \& 
Clark, 2014) it is often limited by the relatively high costs of training and of employing this staff group.

Another way to increase availability and access to treatment for adolescent depression is to develop a treatment that can be delivered by a wider range of professionals in a range of settings. Because this wider group will therefore not be specialist psychological therapists, any new therapy needs to be relatively straightforward and easy to train and to deliver to young people. Given the high risk of self-harm and suicide amongst depressed young people careful supervision is also needed. This case illustration describes one such treatment - Brief Behavioural Activation (Brief BA) - delivered by a non-specialist clinician under supervision. It outlines the theory, method and style of therapy and describes a model of supervised training.

\section{Behavioral Activation for Depression}

Behavioural Activation is an effective treatment for depression in adults (Cuijpers, van Straten, Andersson, \& van Oppen, 2008; Ekers, Richards, \& Gilbody, 2008; Richards et al., 2016). The intervention is based on the behavioural theory of depression which suggests that depression is triggered and maintained by a reduction of reinforcement (and potentially, presence of punishment) for healthy behavior, and/or presence of reinforcement for depressed behavior (e.g. Lewinsohn, 1974; Skinner, 1953).

Although behavioral techniques are used in CBT for depressed adults, cognitive strategies are also included which are typically given greater emphasis. To investigate the relative effectiveness of these individual components, Jacobson et al. (1996) conducted a dismantling study of CBT for adult depression. Contrary to expectations, the behavioral components of CBT (activity scheduling) delivered alone were as effective as full CBT, 
despite excellent treatment adherence in the different conditions and a clear bias favouring full CBT.

This surprising finding led to the increased use of activity scheduling in the treatment of depression, as well as the development of two specific BA therapies. The most comprehensive BA approach for depression (Martell, Addis, \& Jacobson, 2001; Martell, Dimidjian, \& Hermann-Dunn, 2010) includes detailed functional analysis and works both on reducing avoidance behaviors and increasing approach behaviors. Typically the treatment includes 20 sessions or more and includes targeted interventions for wider difficulties such as rumination. BA has strong empirical support for the acute treatment of adult depression (e.g. Dimidjan et al., 2006) and there is evidence that it is effective at reducing relapse (e.g. Dobson et al., 2008).

In comparison, Behavioural Activation Treatment for Depression (BATD: Lejuez, Hopko, \& Hopko, 2001) is a relatively brief and structured intervention with 5 unique content sessions (with up to 5 concept review sessions, so the total treatment usually takes between 6 to 10 sessions). The aim is to identify activities that are consistent with each individual client's values, and support clients to increase valued activities. The concise approach is designed to be delivered by non-specialists (e.g. drug counselors) in both residential and community settings (e.g. Magidson et al., 2011). BATD has been successfully applied to a variety of settings and client groups (e.g. Collado, Castillo, Maero, Lejuez, \& MacPherson, 2014; Gawrysiak, Nicholas, \& Hopko, 2009; Hopko, Lejuez, Lepage, Hopko, \& McNeil, 2003). A ten year revision of the manual was published in 2011 (BATD-R; Lejuez, Hopko, Acierno, Daughters, \& Pagoto, 2011), including a greater emphasis on the treatment rationale and therapeutic alliance. 
A number of meta-analyses have found that the effectiveness of Behavioural Activation approaches is equivalent to CBT in the treatment of depression in adults (Cuijpers et al., 2008; Ekers et al., 2008) and is superior to medication for adult depression (Ekers et al., 2014). Length of treatment and clinician level of training are not associated with treatment outcome (Ekers et al., 2008). A recent non-inferiority RCT with depressed adults found BA delivered by mental health workers was non-inferior to CBT delivered by more expensive CBT therapists (Richards et al., 2016). Importantly, the lower cost of the mental health workers meant delivery of BA was $21 \%$ cheaper than CBT with equivalent outcomes.

\section{Adapting Depression Treatments for Adolescents}

Treatment for adolescent depression tends to be based on adaptations of models developed for the treatment of adults. Adaptions are necessary to accommodate the specific needs of young people. For example, clinicians must consider the developmental stage of the young person and their current cognitive, emotional and social development. Family and systemic issues can support or interfere with treatment and may be associated with the onset and maintenance of the disorder. Young people do not have autonomy and typically rely on their parents to provide practical as well as emotional resources. Additionally, common symptoms of adolescent depression include irritability, poor concentration and low motivation (Orchard, Pass, Marshall, \& Reynolds, 2016) all of which present potential obstacles to treatment.

It is perhaps unsurprising that the effectiveness of psychological therapies for adolescents is modest at best. A meta-analysis of 35 RCTs of psychotherapy for depressed young people indicated a mean effect size of .34 (Weisz et al., 2006). There was no difference between psychotherapies with a cognitive component such as CBT and 'non- 
cognitive' interventions (although this group included a broad range of approaches including attachment based family treatment and group support as well as behavioral therapies).

Behavioural therapy has been adapted for adolescents with depression but there has been limited evaluation of effectiveness. McCauley et al. (2015) randomized 60 depressed adolescents to a 14-session adolescent version of BA (based on Martell and colleagues' model) or to an alternative 'evidence based therapy' (i.e. CBT or Interpersonal Therapy, IPT). Outcomes for BA were equivalent to those for CBT and IPT. BATD has also been adapted for adolescents, with Lejuez and colleagues reporting a successful adolescent case study (Ruggiero, Morris, Hopko, \& Lejuez, 2005). Subsequently, Pass and Reynolds (2014) adapted the most recent version of BATD (BATD-R) by adding material to accommodate the developmental needs of adolescents and including parental involvement (Brief BA).

\section{Brief BA for Adolescent Depression}

Brief BA (Pass \& Reynolds, 2014) retains key elements of BATD-R while incorporating a number of developmental considerations as outlined in Table 1. The content is explicit and structured. Brief BA includes eight, weekly sessions of about an hour. Psychoeducation on depression and the Brief BA approach is provided to young people and their parents before and throughout therapy. Treatment is delivered with the support of session by session adolescent and parent workbooks. The adolescent version of the workbook is used simultaneously as a clinician manual, so the content is transparent and easy to follow (i.e. the clinician and young person work through the adolescent workbook together as the session material), helping scaffold the young person's understanding of Brief BA. A complementary clinician guide and checklist for each session helps support delivery of Brief BA by non-specialist clinicians. 
In Brief BA the focus of the intervention is positive reinforcement (e.g. Lewinsohn, 1974). A simple maintenance formulation (see Figure 1) is used to highlight the young person's current experience of depression and the reduction of positive reinforcement, alongside a future cycle of increased reinforcement and improved mood via behavior change.

[Figure 1 about here]

A key element of Brief BA is the prominence given to identifying the young person's values. Values are described to the young person as 'What matters to you' and 'The way you want to live your life'. The concept of values has been simplified from BATD-R, and made more appropriate to adolescents (see Tables 1 and 2). Values in three main areas are explored: 'Me' (hobbies, physical health, looking after myself), 'Things that matter' (education/work, everyday stuff, the bigger picture) and 'People that matter' (family, friends, boyfriend/girlfriend or important other). The values identified are then used throughout therapy to help select behaviours linked to those values, which are thus more likely to be reinforcing to the young person. Linking activities to values also increases the likelihood of young people engaging in behaviors that are not immediately rewarding, but are reinforced by long-term valued consequences (e.g. revising to pass exams required for a particular career).

During therapy sessions the young person and therapist decide how to schedule valued activities in the young person's day to day life. Sessions are also used to review the impact of activities on the client's mood. Key phrases are used to convey the key message of Brief BA, i.e. 'Doing more of what matters' and 'Getting more out of life' (see Figure 1). Importantly, in contrast to simple activity scheduling, activities that are not in line with the individual's values are only considered if they are mandatory for the young person (e.g. going to school) or if not completing them would cause significant conflict with parents or other 
significant people. Therefore the focus on 'values' also has the benefit of increasing adolescent engagement, by exploring a developmentally salient concept while acknowledging the restrictions placed on them by authoritative figures such as parents and teachers.

To reflect the limited autonomy that adolescents experience compared to adults, Brief BA involves parents in a number of sessions as another developmental consideration. This involvement is secondary to the primary relationship between the young person and the therapist. The role of the parent is to support the young person, to provide positive reinforcement generally (i.e. through smiles, non-verbal behaviours, encouraging comments, etc.) and specifically for valued activity and non-depressed behaviour. Parental involvement is also used to manage differences of opinion about valued activities and to agree structured ways in which parents can help their child engage in valued activities.

Two specific techniques are used with parents and young people, problem-solving and contracting. Problem-solving (added to Brief BA due to the ongoing development of such skills in adolescence) can be used to identify possible solutions to disagreements or conflicts between the young person and parent, as well as considering how to incorporate value-based activities around mandatory activities that are not valued by the young person (e.g. doing valued social activities or hobbies after a day at school). Contracts, an retained feature from BATD-R, are used to establish agreements between the young person and parent (e.g. parent provides a lift to a friend's house, which might be agreed on the condition that the household chores are completed first).

Brief BA includes work for the young person to do between sessions ('homework'). This is discussed at the start of Brief BA, using the analogy of practicing a sport or musical instrument, and highlighting the amount of time in vs outside of sessions (e.g. "we only meet for 1 hour a week, so there are 167 hours a week where we're not together"). However, 
engagement is carefully considered when introducing homework in Brief BA. Activities are designed to be manageable for young people who as a function of their depression are likely to be experiencing low motivation, fatigue, and/or concentration problems. Clinicians are given further information on how to adapt homework tasks to fit the individual client's ability in the clinician guide and in supervision.

The first homework activity in Brief BA is activity monitoring, framed as a 'getting to know you' tool and used flexibly to collect as much information as the young person feels able to record. The young person is asked to record their activities on an activity log then rate them for the level of Achievement (A), Closeness (C) and/or Enjoyment (E) experienced, and if the activity was Important (I) to them personally (this is referred to as 'ACE-I' ratings, adapted from the ACE log; Vivyan, 2014). Ratings can be done with paper and pencil, on a computer, smart phone or in any way the young person feels is manageable. The aim of these ratings is to highlight that a variety of activities can provide the young person with positive reinforcement, not just those they might immediately think of as 'fun'. The rationale is that planning in activities that increase Achievement, Closeness and/or Enjoyment and that are Important to the young person (i.e. in line with their values) maximises the likelihood of them being exposed to stable and diverse sources of positive reinforcement, to ensure longterm improvements in depression symptoms. The rating categories were chosen to highlight how different activities can be reinforcing (e.g. a young person might get a sense of Achievement from cleaning their room, a sense of Closeness from socialising with friends, and Enjoyment from watching a comedy series). Importance ratings were included to enable the young person and therapist to start collecting information about potentially valued activities, and the distinction between what is important to the young person rather than important to others. 
The information gained from the activity log is used in session 3 to identify the young person's values, which form the basis of activity planning. The remaining between session work in Brief BA involves trying out these planned activities, and in the next session reviewing their impact on mood (e.g. "Did you feel better or worse after doing that?") and the feasibility of long-term continuation (e.g. "How easy would it be to do that every week?"). When selecting valued activities, young people are encouraged to consider activities across different life areas, particularly aspects that are currently neglected. With depressed adolescents their 'Me' values that are often neglected for 'Things that matter' like school/college as these are not optional for them. This principle is explained to young people and families with the phrase 'don't put all your eggs in one basket'.

Other key adaptations in Brief BA from the BATD-R manual are outlined in Tables 1 and 2, including how developmental considerations shaped the changes made (e.g. material provided in written and visual form to supplement verbal discussion in session, given the young person's ongoing cognitive development).

[Table 1 about here]

The use of Brief BA with depressed young people has been reported in two previous case studies, which provide further detail on the approach. Pass, Brisco, and Reynolds (2015) outlined the method and reported on acceptability. Pass, Whitney, and Reynolds (2016) described the successful use of Brief BA with a young person with a relatively complex history and active suicidal behaviour during treatment. The current paper reports on a case example where Brief BA was successfully delivered by a non-specialist (a psychology graduate) who had no formal psychotherapy training.

\section{Case Illustration}

\section{Service Context and Clinician Characteristics}


At the time of the therapy, the clinician $(\mathrm{EH})$ was a 26-year-old female psychology graduate. EH was working as a psychology assistant in an outpatient mental health service for children and adolescents. The service was part of the UK National Health Service and thus was open to anyone living within the catchment area and was free to access. This was based in a large town (population approximately 155,000) in the south of England. The clinician's primary role in the service was to deliver guided self-help CBT to parents of children who had been diagnosed with an anxiety disorder (Thirlwall et al., 2013). In her usual role she was supervised by a clinical psychologist. She had no experience of psychological interventions with depressed adolescents.

\section{Client Background and Characteristics}

'Emma' (a pseudonym) was a 16-year-old Black-British girl. She lived with her mother and reported having a very close relationship with her. Emma was referred to the Child and Adolescent Mental Health Service (CAMHS) by her family physician due to difficulties with low mood, deliberate self-harm and suicidal ideation.

Emma had experienced a number of significant losses preceding her referral to CAMHS. Emma's father had died from cancer when she was 13 years old, and her paternal grandmother died when Emma was 14. Her mother started a new relationship when Emma was 15 and at the same time, Emma started experiencing significant friendship difficulties. Emma had received grief counselling after her father died and had received counselling sessions a year before her referral. She said that it had been helpful to talk about her feelings but she and her mother agreed that counselling had not led to any positive change. Emma's mother felt that Emma would benefit from a more formal therapeutic approach, and Emma agreed. Emma was not taking any medication for depression at the time of assessment and did not start any medication during treatment. 
This case example was chosen as it was the first time that a non-specialist delivered Brief BA to a young person with depression. The family gave consent to be audio recorded in session, and to share their experience with others.

\section{Assessment}

\section{Diagnostic Interview}

An abbreviated diagnostic clinical interview was completed with Emma and her mother by a qualified clinical psychologist as part of standard practice in the service. This interview involves exploration of symptoms to establish presence of mood and anxiety disorders in young people, based on DSM-IV criteria (American Psychiatric Association, 1994). Emma was seen alone for part of the interview, as per standard practice.

Emma described low mood, lethargy, trouble sleeping, feelings of guilt, and difficulty concentrating. She reported occasional suicidal thoughts but said that these were primarily a passive idea of wishing she was no longer here rather than specific plans to kill herself. Emma reported no intent or plans to act upon these thoughts. Triggers for suicidal ideation included conflict with her mother or peers. Emma had been experiencing suicidal thoughts most days, but reported that the frequency and intensity of these thoughts had recently reduced. She reported that she had self-harmed by cutting her forearms and thighs, but not in the two months before the assessment. A safety plan was devised with Emma and her mother, which identified warning signs/ triggers for harming herself (conflict at school or at home) and ways of coping (letting her mother know how she was feeling, chatting to a friend, or cuddling her pet rabbit). It was agreed that when Emma was feeling low at night she could go to her mother's room. Emma and her mother were given information about sources of support and emergency procedures. 
Emma did not attend school every day and was not seeing her friends as often as she used to. She found it difficult to get up in the morning and this was a source of conflict between Emma and her mother. Emma reported anxiety, particularly in social situations, and occasional panic attacks. She felt that the symptoms of anxiety developed after the symptoms of depression, and that they would be easier to deal with if her mood improved.

Based on Emma and her mother's responses to the abbreviated diagnostic interview at assessment, Emma met criteria for moderate Major Depressive Disorder (MDD) with six symptoms: Depressed mood/irritability, fatigue, sleep disturbance (initial insomnia), excessive feelings of guilt, cognitive difficulties and suicidal ideation. Emma did not meet criteria for any anxiety, or other mental health disorders.

Emma and her mother also completed the following questionnaires which are routinely used in Child and Adolescent Mental Health services in England, in line with the Routine Outcome Measures (ROMs) approach.

Revised Child Anxiety and Depression Scale: Child and Parent versions (RCADS, RCADS-P; Chorpita, Yim, Moffitt, Umemoto, \& Francis, 2000)

These are 47-item questionnaires that assess anxiety and low mood in young people aged 8 to 18 years. There are subscales for depression and five anxiety disorders (social phobia, panic disorder, separation anxiety, generalized anxiety, and obsessive-compulsive disorder). The full RCADS and RCADS-P were completed at the initial diagnostic interview and at the final treatment session (session 8). The self and parent report depression subscales were completed at the start of every BA session. An additional question ('I thought about killing myself'/'My child thought about killing themselves') was added to monitor risk. The RCADS has good construct validity (Chorpita et al., 2000), high internal consistency (Chorpita, Moffitt, \& Gray, 2005) and test-retest reliability (Chorpita et al., 2000). The 
RCADS-P has also shown good psychometric properties (Ebesutani, Bernstein, Nakamura, Chorpita, \& Weisz, 2010).

At assessment, Emma's RCADS indicated she was in the clinical range for depression, panic and separation anxiety. Emma's mother's RCADS-P was similar but also indicated social anxiety.

Outcome Rating Scale (ORS; Miller \& Duncan, 2000)

The ORS was used to assess Emma's functioning. On the ORS young people and their parent are asked to rate the young person's functioning in four key areas: Individual, Interpersonal, Social and Overall. The ORS has high internal consistency and test retest reliability, and good concurrent validity with other treatment outcome measures (Duncan, Sparks, Miller, Bohanske, \& Claud, 2006). The ORS was completed and reviewed every session to consider progress, reflect on agreement or differences in scores between adolescent and parent report, and to highlight particular areas of difficulty.

Goals

Treatment goals were agreed in the first session of Brief BA. Emma initially found it difficult to identify any goals; with help from the clinician and her mother she identified 'Meeting up with friends once a week' and 'Getting more exercise (play tennis several times a week)'. She rated herself as $0 / 10$ towards achieving these goals at session 1 .

\section{Formulation}

At her intake assessment interview the assessing clinician (HW) developed a behavioral formulation with Emma and her mother. It was hypothesized that a series of significant losses had led to reduced positive reinforcement and low mood. Low mood was then maintained by Emma's lack of activity and social withdrawal. Emma's low activity 
levels also increased her sense of lethargy and also increased her anxiety about the avoided situations. Emma and her mother strongly identified with this formulation. Emma attributed some recent improvements in mood to re-engaging with activities. Treatment options were discussed with the family (including CBT, medication, Brief BA) as part of shared decision making and Brief BA was chosen collaboratively.

\section{Brief BA intervention}

\section{Course of Brief BA Treatment and Use of Supervision}

Emma attended eight, weekly one hour sessions. Emma's mother attended sessions 1, 6 and 8 . As this was a training case delivered by a non-specialist with limited clinical experience, the case was closely supervised. Supervision was scheduled before the first session and after every treatment session. Supervision was typically 1 to 1.5 hours, in a group setting (with 1-2 other clinicians delivering Brief BA) and was led by a qualified clinical psychologist (HW). Standard agenda items included: reviewing routine outcome measures, risk monitoring, presenting an audio clip of the previous session with a BA related question, and discussing the clinician's own reflections on using BA.

Pre-session Training and Supervision

Before starting Brief BA with Emma, the clinician reviewed the treatment manuals, session checklists and read Emma's referral and assessment notes. Before session 1 the clinician and supervisor (who was also the assessing clinician) discussed the case. They reviewed the provisional behavioural formulation that had been developed with Emma and her mother. 
Brief BA sessions with Emma began three weeks after the assessment. Details of Brief BA session content and how this differs from the BATD-R protocol are provided in Table 2. The clinician followed the standard session plan as outlined.

[Table 2 about here]

\section{Themes from Brief BA Therapy and Supervision}

Themes emerging from both the novice Brief BA clinician's sessions with Emma, and discussion of these in supervision, are outlined below.

Theme 1: The importance of the clinician's own understanding of the Brief BA rationale

As a novice Brief BA clinician, EH had limited knowledge of behavioral theory. No prior knowledge is assumed in Brief BA training and therapy materials, and attention is given to developing both competence and confidence in the approach. The Brief BA training materials and practical workbooks were key to the clinician's learning about the approach. A supervision session was also arranged before the first Brief BA session. During this session the clinician practiced, using role play, how to explain the Brief BA rationale to Emma and her mother. Below is an excerpt from Emma's first session, where the clinician introduces the rationale to provide socialisation to the Brief BA model.

Clinician: Okay, so this treatment approach which is called Brief BA is focused on the idea that your ability to feel better has a lot to do with what you are doing on a day to day basis. It's about identifying your patterns of behavior, so what you are doing day to day, and trying to increase the helpful or important activities, which differs from person to person. So it's about trying to identify that and decreasing the unhelpful activities that aren't going to bring you much. Interestingly often with people experiencing depression, what they think is important and what they are 
actually doing is quite different. So people have an awareness of what they would like to do and what is important to them but they are actually finding it really difficult to do those things.

It is essential that all Brief BA clinicians have a solid understanding of the Brief BA rationale, are confident that Brief BA can significantly improve young people's depression symptoms, and convey this in their description of the model. For EH her background in CBT based interventions meant she was sympathetic to the treatment model. Her lack of experience working with depressed adolescents also meant she did not have alternative therapeutic approaches she typically used with this client group and thus did not have to 'unlearn' techniques or alternative treatment models. However, as a novice Brief BA clinician she initially lacked confidence in explaining the rationale to families, so found role playing this discussion very helpful.

\section{Theme 2: Engagement of depressed adolescents in therapy}

Engaging depressed adolescents in therapy is not easy. Engagement is an important topic for discussion in supervision, particularly with novice clinicians. To help identify early barriers to engagement and to build collaboration in Brief BA the young person is explicitly asked for feedback at every session. At the beginning of session 2, EH and Emma reviewed the first session. Emma reported that "It was a little bit scary at first. I'm always like that at the beginning though but then I get relaxed and am fine with it". The clinician empathized with Emma's worries about meeting someone new in a new situation. Emma responded positively to this, and became more relaxed as the session progressed.

Non-completion of between-session work (and discussion of this) can often be a barrier to engagement with young people. The transcript below shows the discussion in 
session 2, in response to Emma not completing the BA homework (the activity log) from session 1.

Clinician (C): Shall we have a look at the activity log and see how you are getting on with that?

[Pause]

Emma (E): That went completely out of my mind [apologetic tone].

C: That's okay! Okay so what do you think was difficult about it- was it that you forgot all about it?

E: I mean I did start doing it as soon as I went back to school, but I forgot to save it on my phone so it wiped off and it just completely wiped itself out of my memory.

C: Ah okay, so you did start doing it and managed to have a think about it, but it didn't save?

E: Yeah.

C: Okay, do you think there is anything you could do this week that might help you to remember?

[Pause]

C: I'm just having a think as well...

[Pause]

$\mathrm{C}$ : Is there any way you could set a reminder on your phone?

E: Yeah but I'm not quite sure how to do it. 
C: Not sure how to do that, okay.

E: My memory is like one of my weakest points.

$\mathrm{C}$ : Yeah, so that might be something we can think about. Was there anything else that made it difficult for you to complete it or was it just that you completely forgot?

E: I just forgot.

C: Okay so it would have felt okay for you to try if you had remembered...

\section{[Client nods]}

C: Perhaps we could think back to what happened this week- would that be okay to try?

E. Mmhm [nods in agreement].

Following this discussion, the EH and Emma recorded Emma's activities on a monitoring form together, to reinforce the benefits of recording this information.

The clinician brought the issue of homework non-completion to supervision, as directed in the Brief BA clinician guide. Difficulties with completing between session activities are very common and this can be helpful for trainee clinicians to hear. Peer supervision also provides opportunities to share experiences and to offer and discuss possible solutions. After supervision EH reflected that it had been important to hear her supervisor normalizing the fact that clients often have problems completing homework. This made her less anxious that her lack of experience had been the cause of the difficulty. She identified that she could have explored the potential benefits of the activity log with Emma more explicitly in the first session. She had also found it useful to discuss the homework difficulty 
openly and collaboratively in the session. This discussion also gave opportunities for the clinician to model flexibility and to reinforce the young person's efforts.

In the following session, Emma brought in her completed activity log (see Video Clip 1) and started engaging more actively in work between sessions. Emma reported that she had received support in her Math lesson at school, and rated this lesson as high for ACE-I. She also identified that she was spending most of the weekends in her bedroom sleeping, playing video games or on social media (activities low on ACE-I ratings).

[Video clip 1 about here: Reviewing Activity Log]

Another challenge to engaging young depressed people in Brief BA is balancing fidelity to the manual with flexibility within sessions (Cassar et al., 2016; Kendall, Chu, Gifford, Hayes, \& Nauta, 1999). Brief BA is designed to be delivered by non-specialist clinicians so is very structured. It must also be delivered sensitively and flexibly to ensure that young people remain engaged and the therapeutic alliance protected. As sessions progressed, the clinician observed that Emma was not typically using her activity log between sessions but that she was increasing her valued activities. In supervision Emma's overall progress was reviewed. Emma was engaging in treatment, increasing her activities and her symptoms were beginning to reduce. Given that the ultimate aim of Brief BA is to increase positive reinforcement it was agreed that keeping the activity log was not critical if progress was being made without this. Without this supervision, a non-specialist clinician may feel the need to stick rigidly to the manual which could compromise the therapeutic alliance and engagement in Brief BA.

Theme 3: Exploring the young person's values and translating these into valued activities 
Values can be an unfamiliar concept for many clinicians, and learning how to explore these in a validating, engaging way with a young person is essential. Values represent a key difference in Brief BA (and BATD) from simple activity scheduling, as these determine what kind of activities should be scheduled (and which activities may need to be reduced in order to facilitate this). In this case example, EH attended group supervision before her first session with Emma and found it useful to hear specific examples about how other clinicians had explored values with other young people.

Video 2 illustrates how the concept of 'values' was described to Emma by EH and the values Emma was able to identify in this discussion. The life areas and values identified by Emma across Brief BA as a whole are summarized in Table 3.

\section{[Video clip 2 about here: Identifying Values]}

\section{[Table 3 about here]}

Following exploration of Emma's values, the clinician used supervision to reflect on how the discussion about values felt very collaborative, and how she was pleasantly surprised at how easily Emma was able to identify her own values. This is a common theme in Brief BA supervision. Non-specialist clinicians often worry about eliciting values from young people, but are generally pleased to find how straightforward this can be when drawing on information collected by activity monitoring.

After a young person identifies their personal values, these are translated into meaningful and achievable activities. Emma was able to identify several activities that met her values. For example, for Emma's value of 'getting a good grade in Art' she decided to practice drawing at home, try drawings things that were more difficult and out of her 'comfort zone', and to get started on her art book at school. Emma also decided that she 
would like to try spending more time with her mother's partner 'John' (a pseudonym). She suggested that they could play on the game console together - this met her value of 'helping family with their goals' (one of John's goals was to get closer to Emma).

Linking values and activities can also be done by working backwards from activities that the young person has rated highly as 'important', and exploring why that behaviour was important for them. This can highlight other activities that fit the same value. For example, when reviewing the previous week in session 4, Emma reported that she had felt less tired during the day, more tired in the evening and had been sleeping better at night since the last session. The clinician encouraged Emma to consider anything she had been doing differently over the past week. Emma realized that she had been playing bench ball in her PE lessons at school and that this had got her "up and moving", in line with her value of 'doing sports to keep fit'. This insight helped Emma to strengthen the link between her values and possible activities (e.g. actively engaging in other PE lessons as school, practice dance for longer at home, find a tennis club), and link her activities to her mood.

Supervision was used to reflect on positive and challenging elements of delivering Brief BA for the first time. EH had anticipated more difficulty linking values and activities in sessions 4 than she had encountered. Emma had engaged with treatment and started to make changes outside of the treatment sessions. Acknowledging these changes in supervision helped EH feel more confident both in her own ability to deliver the treatment and in the treatment model. Emma's progress and engagement with treatment had a positive effect on the clinician's sense of competence using Brief BA and working with depressed adolescents.

\section{Theme 4: Using Routine Outcome Measures and Risk Monitoring every Session}

Routine Outcome Measures (ROMs) are embedded within Brief BA, and clinicians are expected to use these in every clinical and supervision session. This is for a number of 
reasons. Firstly, feedback from young people and families on using ROMs is very positive as long as the data are collected in a collaborative way, considered within the context of other clinical information, and used practically in sessions rather than a 'tick box' exercise (Stasiak et al., 2013). Secondly, ROMs provide regular information for clinicians to track progress and help identify specific difficulties the client might not otherwise mention spontaneously (e.g. sleep problems, specific school difficulties). ROMs are particularly valuable as a way of monitoring risk and of making this a routine part of treatment. Thirdly, routine outcome monitoring are useful in supervision. ROMs help link symptomatic improvement to increased activities, monitor client's progress and highlight lack of change and importantly, deterioration.

The rationale for using ROMs must be carefully explained to young people and parents. They must always be reviewed with the young person and/or parent in the treatment session. This means that time must be allocated to complete questionnaires (e.g. before the session begins) and to review them each week. In this case example ROMs were reviewed with Emma every week and often prompted further discussion about changes that had led to improvements or deterioration. For example, in session 7 Emma reported worsening low mood; this was reflected in an increase on the RCADS depression subscale score from 7 (at session 6) to 10 in session 7 (see Figure 2). Similarly, Emma's ORS score dropped from 28 to 21.1 in the same week (see Figure 3). Emma identified problems with her friends as a possible trigger for her lowered mood, and problem-solving was used in the session to consider ways to address this.

Many depressed young people (including Emma) experience co-morbid anxiety symptoms. Brief BA specifically focuses on key symptoms of depression, so anxiety symptoms are not directly addressed. Where they are present at assessment they are monitored using the RCADS. Where anxiety is interfering with Brief BA, work on this can 
be incorporated. For example, if social anxiety prevents a young person engaging in valued activities, this can be considered within a behavioral context (e.g. using problem-solving and contracts to break the activity down into smaller steps and elicit support). However, supervision is essential to consider the need to address anxiety symptoms within Brief BA as this is not part of the standard protocol.

It is also possible that anxiety symptoms do not affect progress in Brief BA, and may reduce in line with increased valued activity without any targeted anxiety work. For Emma, although her anxiety symptoms were above the clinical threshold on the RCADS at the start of Brief BA, she did not meet criteria for an anxiety disorder as the symptoms were not significantly impairing her functioning. Although her anxiety symptoms remained in the clinical range at the end of Brief BA, Emma reported making progress in a number of areas (e.g. paying for shopping herself and other tasks that increased her sense of achievement and autonomy) and symptoms were not affecting her functioning; therefore further anxietyspecific intervention was not indicated.

Adolescents with depression are at high risk of self-harm and suicidal ideation. In Brief BA risk assessment is embedded into routine outcome monitoring. In this case, risk assessment in session 3 highlighted that Emma had engaged in self-harm in the previous week, following an argument with her mother. Emma told the clinician that she had cut her arm with a pair of scissors, and showed EH the marks on her arm (these were superficial and were healing well). Emma had told her mother and a close friend about the self-harm. In line with standard service and Brief BA therapy procedures, the clinician asked Emma for further details about the self-harm and the safety plan was reviewed.

As directed in the Brief BA clinician guide, EH brought the risk issue to supervision. The routine inclusion of the risk screening question on the RCADS depression subscale had 
prompted the conversation about self-harm. EH reflected that there had been a lot to cover in the session, and more time would have been useful to review the safety plan in more detail with the involvement of Emma's mother. Where additional time is needed to manage risk the clinician guide advises adding extra sessions (Pass, Brisco, Hodgson, \& Reynolds, 2015; Pass \& Reynolds, 2014). In supervision it was agreed that EH would continue to discuss risk with Emma at every session and that this information would be shared with Emma's mother if necessary.

Theme 5: Involving parents in treatment for adolescent depression

Balancing the contributions and involvement of young people and their parents in therapy sessions is a core skill in Brief BA. In Brief BA parents are routinely invited into sessions (1, 6 and 8). There is flexibility to respond to client preference and the manual does not specify exactly when in the session parents are included, or for how long. Session 1 with the parent involves psycho-education about the Brief BA model and discussion about the use of the parent manual and the role of reinforcement (in general and from parents). This is discussed to ensure that parents understand what their child will be working on in sessions that the parent does not attend, and to engage the parent in the aims and style of therapy.

Session 6 is typically a joint session in which the clinician, parent and young person review progress. This session is also used to identify ways the parent can support their child, establish contracts, identify conflict or obstacles and use problem-solving techniques to address these. Contracts are a specific way to elicit emotional and practical help from parents in a manner acceptable to the young person. Often parents are desperate to help their child, but may unintentionally reinforce only depressed behaviour. They also may not recognise the tasks their child requires help with, and those they are able to do (and may well wish to do) independently. 
Emma attended the main part of session 6 alone and her mother attended for the final 20 minutes. When the clinician introduced the idea of contracts to Emma in session 6, Emma identified that she needed help to improve her dancing. She suggested that her mother could help by giving feedback and helping her find a space to practice at home. This was received well by Emma's mother, who was pleased to agree a specific, practical way she could support Emma in her treatment.

Next, the clinician introduced the technique of problem-solving with the support of a visual flow-chart. Emma and the clinician used the technique to discuss a problem Emma identified - that she did not feeling confident about her drawing. Emma engaged well with this practical task, and identified numerous solutions including 'watch other people draw', 'look at other people's art styles', and 'use colors'. Emma decided that the best solution was to 'watch other people draw' via videos online and she planned to try this at the weekend.

In Brief BA over-involvement of parents in the session can make it difficult to ensure that the young person receives positive reinforcement for their own actions, and may undermine their sense of autonomy and independent problem-solving. In session 6 the clinician observed that Emma became much less active when her mother was in the room for the last part of the session. She highlighted a segment of the session to demonstrate this and brought this to supervision.

Clinician (C): I don't know if your school ever hires out... some schools hire out halls?

Mother (M): I've never thought of asking the school actually.

Emma (E): I doubt they would. 
M: Yeah but the thing is if I spoke to...because I've got to make an appointment to see 'Miss Cook' [pseudonym]- the head of year, so that we can have a catch up to see how things are going. And if I mention it to her that it could be good for the things you are doing with CAMHs, and she can organize it so that somebody would be able to stay after school with you in the sports area. Perhaps you could do it on the night when there is a late bus.

E: I could get 'Jane' [pseudonym] to do it with me- she gets really competitive.

M: Yes Jane would be good actually because she would push you. She would make you puff [laughs]. And then you know I could give Jane a lift home.

C: Okay that's an idea then isn't it?

M: Yeah, perhaps you and Jane could organize this yourself then.

E: That would go with meeting up with friends as well.

C: Yeah that would help towards both of them.

M: Yeah it would combine the two! How about we give you a challenge that you and Jane organize that between you?

E: Okay.

M: Yeah, see if you can do it by the end of the week.

E: Mmhm [in agreement].

C: Great, okay that's a good idea.

Ways of structuring the session, asking questions and supporting Emma in sessions where her mother was present were discussed in supervision, as well as seeking feedback 
from Emma as to how she experienced this input from her mother. In future joint sessions, the clinician was careful to elicit Emma's views and opinions to ensure a balance between her own and her mother's input.

\section{Theme 6: The reinforcing role of the clinician}

Throughout Brief BA training and in supervision, the key role of the clinician as a source of positive reinforcement (and potential source of punishment) is considered. For example there is potential for young people to feel 'punished' when they report that they have not managed to complete agreed homework tasks. This experience can remind them of difficulties at school where not completing homework is almost always followed by some form of explicit punishment. In Brief BA the role of the clinician is to maintain a validating, empathic stance and to reinforce any non-depressed behaviour (no matter how small). This is illustrated at the start of video clip 2, where Emma apologised for the limited completion of her activity log. In response, instead of focusing on what was not achieved, the clinician provided reinforcement for Emma's effort and praised the work she had completed.

Because clinicians typically become a significant source of reinforcement to their client, endings in therapy represent a potential loss. Emma engaged well in Brief BA and appeared to experience the relationship with the clinician as particularly rewarding. The clinician also reported many occasions when she had offered verbal reinforcement during sessions and that this was received positively by Emma. Given this, Emma's concerns about ending therapy were discussed in supervision and ways of preparing to end therapy were considered. As a result the clinician added 'ending Brief BA' to the agenda in every remaining session. The clinician explored with Emma the important changes that she had made and emphasised the independent nature of these. She noted that in later sessions Emma started to praise herself for increasing her activity level (providing her own positive 
reinforcement), and that Emma's mother was keen and able to help support Emma when therapy ended.

In session 8, although considerable progress was reported by both Emma and her mother (and indicated by their ORS scores), both also reported an increase in Emma's depressive symptoms. Emma reported concerns about ending therapy, and when providing written feedback to the question 'what did you like least about BA?', she wrote 'I'm afraid when it ends I might go back to how I was'. Emma reported that Brief BA had "definitely helped and hopefully my mood will get better. But if it doesn't then I'm not sure. I have got used to coming so it will be weird to just stop".

\section{Outcome of Brief BA}

\section{Treatment outcome}

At the review session one month after the end of Brief BA therapy, Emma described sleeping better and having more energy. She was attending school regularly and finding it easier to wake up in the mornings. She no longer experienced suicidal thoughts and had not self-harmed since session 3.

At the end of treatment Emma's self-reported questionnaire scores showed improvement. Both her RCADS depression subscale and ORS scores showed reliable and clinically significant improvement, moving from a clinical range at session 1 to a normal range by review (see Figure 2). The full RCADS was completed by Emma and her mother again at session 8 to assess anxiety symptoms. Emma and her mother reported that Emma's panic and separation anxiety scores were still in the clinical range. Emma's mother (but not Emma) reported that her social anxiety symptoms were now in the normal range.

[Figure 2 about here] 
Emma's goals were reviewed through the course of Brief BA. At the review session, Emma rated her progress towards both of her goals as 9/10. Emma explained that she had been doing more exercise, meeting up with her friends every few weeks outside of school and keeping in contact with friends online.

Emma was very positive about therapy. She reported that Brief BA "has helped me to work on things I had never thought to work on before" and "has lifted my mood so much". Emma's mother noticed that Emma seemed happier and her overall sense of well-being had improved. Emma's mother reported that Brief BA "made everything seem more positive". When thinking about the end of Brief BA treatment Emma said "I was worried I would go back to how I was feeling before, and was almost convincing myself that this would happen. However, recently I have been thinking about it differently and am now much happier and confident I will be okay". The clinician, supervisor, Emma and her mother all agreed that Emma would be discharged from the service. She has not been re-referred in the year since treatment, suggesting improvements were sustained following the end of Brief BA.

\section{Discussion}

It is important to consider supervision as well as clinician requirements in Brief BA. While for this first case the clinician had high frequency and duration of supervision, this was then tapered so supervision for further cases was provided in a group format (with 1-2 other BA clinicians), for $1.5 \mathrm{hrs}$ fortnightly. The investment of greater supervision for the first case allowed closer monitoring of Brief BA skill development and risk management, enabling a reduced supervision workload for future cases. This model has worked well in our service, and is one we have rolled out with other novice clinicians to good effect. We have also begun to video Brief BA supervision sessions as routine practice, so recurring topics and 
discussions can be monitored, as well as providing an additional resource for novice Brief BA clinicians.

In the course of delivering Brief BA, the clinician was able to provide feedback on the experience of training and learning. As a result supplementary training materials were developed to provide additional guidance on some of the issues this clinician encountered or learned from through supervision (e.g. a frequently asked questions section in the clinician guide including 'What do I do if a young person has not completed their activity log?', and 'How do I manage the ending of Brief BA sessions?'). We have also implemented an experiential approach to Brief BA training for new clinicians, where everyone is asked to try out Brief BA activities i.e. activity monitoring and identifying values, before starting clinical cases. These Brief BA tasks are discussed in group supervision (without detailed personal disclosure) to help clinicians identify some common barriers and challenges to engaging in Brief BA.

EH's background and understanding of CBT based interventions for anxiety meant she had an existing orientation towards cognitive and behavioral approaches. It is likely that this helped with her learning of Brief BA treatment delivery and this may be more challenging to clinicians who have other therapeutic orientations. For example, the structure of Brief BA might be challenging to therapists who have experience working in humanistic or psychodynamic therapy modalities. The structure of the Brief BA manual and addition of supplementary training and therapy resources are designed to help with this transition, and supervision (with use of session material) is key to ensuring adherence to the model. Research is needed to evaluate the efficacy of Brief BA training programs and fidelity to the approach, particularly for clinicians more aligned with other therapeutic approaches. 
Brief BA is a promising treatment for adolescent depression and may offer an alternative to more complex and lengthy therapies for adolescents with depression if the approach is supported by further research. There are also techniques and methods that could be explored further as part of Brief BA. For example, using technology has great potential, especially as it may increase engagement with adolescents used to interacting with such tools. These may include smart phone apps, electronic versions of therapy workbooks, and personal activity trackers. Many of these can also be shared so that progress can be tracked by clinicians and clients simultaneously.

Another consideration is whether Brief BA could be delivered within a modular treatment approach, where additional modules for anxiety (e.g. graded exposure) are added if necessary. For this case, although anxiety symptoms did not remit during Brief BA, they did not impact on treatment or functioning so additional input was not deemed necessary by the clinical team, or the young person or mother. However, this could be a useful addition for many young people, so further investigation of this would be useful. Brief BA could also be incorporated as a focused piece of work within a longer term supportive therapy, as the two are not mutually exclusive. Top-up Brief BA sessions may also be useful to help prevent relapse (if the service context allows), and could be explored further in future research.

\section{Conclusion}

The current case example illustrates the successful delivery of Brief BA by a nonspecialist mental health worker, with minimal previous experience working with adolescents. During training regular supervision that focuses specifically on the Brief BA model and addresses trainees concerns about competence is important to maintain adherence to the approach and to problem-solve any difficulties with engagement. Brief BA is a promising brief intervention for adolescent depression that may be effective if delivered by non-expert 
clinicians. This approach could be provided as part of a comprehensive stepped care model and thus increase the availability of treatment and improve the accessibility of psychological therapies for this at risk group. 


\section{References}

American Psychiatric Association. (1994). Diagnostic and statistical manual of mental disorders: DSM- IV. (4th ed). Washington, DC: American Psychiatric Association.

Avenevoli, S., Swendsen, J., He, J., Burstein, M., \& Merikangas, K. R. (2015). Major depression in the national comorbidity survey-adolescent supplement: Prevalence, correlates, and treatment. Journal of the American Academy of Child and Adolescent Psychiatry, 54, 37-44. doi: 10.1016/j.jaac.2014.10.010.

Birmaher, B., Brent, D., \& AACAP Work Group on Quality Issues (2007). Practice parameter for the assessment and treatment of children and adolescents with depressive disorders. Journal of the American Academy of Child \& Adolescent Psychiatry, 46, 15031526.

British Association of Behavioural and Cognitive Psychotherapies (2012). Minimum training standards for the practice of Cognitive Behavioural Therapy (CBT). Retrieved from: http://www.babcp.com/Accreditation/Minimum-Training-Standards.aspx

Cassar, J., Ross, J., Dahne, J., Ewer, P., Teesson, M., Hopko, D., \& Lejuez, C. W. (2016). Therapist tips for the brief behavioural activation therapy for depression-revised (BATDR) treatment manual practical wisdom and clinical nuance. Clinical Psychologist, 46-53. doi:10.1111/cp.12085

Cash, S. J., \& Bridge, J. A. (2009). Epidemiology of youth suicide and suicidal behavior. Current Opinion in Pediatrics, 21(5), 613-691. doi: 10.1097/MOP.0b013e32833063e1.

Chorpita, B. F., Yim, L., Moffitt, C., Umemoto, L. A., \& Francis, S. E. (2000). Assessment of symptoms of DSM-IV anxiety and depression in children: A revised child anxiety and 
depression scale. Behaviour Research and Therapy, 38, 835-855. doi: 10.1016/S00057967(99)00130-8.

Chorpita, B. F., Moffitt, C. E., Gray, J. A. (2005). Psychometric properties of the revised child anxiety and depression scale in a clinical sample. Behaviour Research and Therapy, 43, 309-322. doi: 10.1016/j.brat.2004.02.004.

Collado, A., Castillo, S. D., Maero, F., Lejuez, C. W., \& MacPherson, L. (2014). Pilot of the brief behavioral activation treatment for depression in Latinos with limited English proficiency: Preliminary evaluation of efficacy and acceptability. Behavior Therapy, 45, 102-115. doi:10.1016/j. beth.2013.10.001.

Cuijpers, P., van Straten, A., Andersson, G., \& van Oppen, P. (2008). Psychotherapy for depression in adults: A meta-analysis of comparative outcome studies. Journal of Consulting and Clinical Psychology, 76, 909-922. doi: 10.1037/a0013075.

Dimidjian, S., Hollon, S. D., Dobson, K. S., Schmaling, K. B., Kohlenberg, R., Addis, M., . . . Jacobson, N. S. (2006). Randomized trial of behavioral activation, cognitive therapy, and antidepressant medication in the acute treatment of adults with major depression. Journal of Consulting and Clinical Psychology, 74, 658-67. doi: 10.1037/0022-006X.74.4.658.

Dobson K.S., Hollon, S.D., Dimidjian, S., Schmaling, K.B., Kohlenberg, R.J., ...Jacobson, N.S. (2008). Randomized trial of behavioral activation, cognitive therapy, and antidepressant medication in the prevention of relapse and recurrence in major depression. Journal of Consulting and Clinical Psychology, 76, 468-77. doi: : 10.1037/0022006X.76.3.468. 
Duncan B. L., Sparks J., Miller S. D., Bohanske R., \& Claud D. (2006). Giving youth a voice: A preliminary study of the reliability and validity of a brief outcome measure for children, adolescents, and caretakers. Journal of Brief Therapy, 5, 66-82.

Ebesutani, C., Bernstein, A., Nakamura, B. J., Chorpita, B. F., \& Weisz, J. R. (2010). A psychometric analysis of the revised child anxiety and depression scale-parent version in a clinical sample. Journal of Abnormal Child Psychology, 38(2), 249-260. doi: 10.1007/s10802-009-9363-8.

Ekers, D., Richards, D., McMillan, D., Bland, J. M., \& Gilbody, S. (2011). Behavioural activation delivered by the non-specialist: Phase II randomised controlled trial. The British Journal of Psychiatry, 198(1), 66-72. doi: 10.1192/bjp.bp.110.079111.

Ekers, D., Richards, D., \& Gilbody, S. (2008). A meta-analysis of randomized trials of behavioural treatment of depression. Psychological medicine, 38(5), 611-623. doi: 10.1017/S0033291707001614.

Ekers, D., Webster, L., Van Straten, A., Cuijpers, P., Richards, D., \& Gilbody, S. (2014). Behavioural activation for depression; an update of meta-analysis of effectiveness and sub group analysis. Plos One, 9(6), e100100. doi: 10.1371/journal.pone.0100100.

Fergusson, D.M., Boden, J. M., \& Horwood, L. J. (2007). Recurrence of major depression in adolescence and early adulthood, and later mental health, educational and economic outcomes. The British Journal of Psychiatry, 191, 335-342. doi: 10.1192/bjp.bp.107.036079.

Gawrysiak, M., Nicholas, C., \& Hopko, D. R. (2009). Behavioral activation for moderately depressed university students: Randomized controlled trial. Journal of Counseling Psychology, 56, 468-475. DOI: 10.1037/a0016383. 
Hopko, D. R., Lejuez, C. W., Lepage, J. P., Hopko, S. D., \& McNeil, D. W. (2003). A Brief Behavioral Activation Treatment for Depression a randomized pilot trial within an inpatient psychiatric hospital. Behavior Modification, 27, 458-469. doi: $10.1177 / 0145445503255489$.

Jacobson, N. S., Dobson, K. S., Truax, P. A., Addis, M. E., Koerner, K., Gollan, J. K., . . . Prince, S. E. (1996). A component analysis of cognitive-behavioral treatment for depression. Journal of Consulting and Clinical Psychology, 64, 295-304. doi: 10.1037/0022-006X.64.2.295.

Johar, M., \& Truong, J. (2014). Direct and indirect effect of depression in adolescence on adult wages. Applied Economics, 46(36), 4431-4444. doi: 10.1080/00036846.2014.962227.

Kendall, P. C., Chu, B., Gifford, A., Hayes, C., \& Nauta, M. (1999). Breathing life into a manual: Flexibility and creativity with manual-based treatments. Cognitive and Behavioral Practice, 5, 177-198. doi:10.1016/S1077-7229(98)80004-7.

Layard, R., \& Clark, D. M. (2014). Thrive: The power of evidence-based psychological therapies. London, UK: Penguin.

Lejuez, C. W., Hopko, D. R., \& Hopko, S. D. (2001). A brief behavioral activation treatment for depression: Treatment manual. Behavior Modification, 25, 255-286. doi: $10.1177 / 0145445501252005$.

Lejuez, C. W., Hopko, D. R., Acierno, R., Daughters, S. B., \& Pagoto, S. L. (2011). Ten year revision of the brief behavioral activation treatment for depression : Revised treatment manual. Behavior Modification, 35, 111-161. doi: 10.1177/0145445510390929. 
Lewinsohn, P. (1974). A behavioral approach to depression. In R. J. Friedman \& M. M. Katz (Eds.), Psychology of depression: Contemporary theory and research (pp. 157-185). Oxford, UK: John Wiley.

Magidson, J. F., Gorka, S. M., MacPherson, L., Hopko, D. R., Blanco, C., Lejuez, C. W., \& Daughters, S. B. (2011). Examining the effect of the Life Enhancement Treatment for Substance Use (LETS ACT) on residential substance abuse treatment retention. Addictive Behaviors, 36, 615-623. doi: 10.1016/j.addbeh.2011.01.016.

Martell, C. R., Addis, M. E., \& Jacobson, N. S. (2001). Depression in context: Strategies for guided action. New York: Norton.

Martell, C. R., Dimidjian, S., \& Hermann-Dunn, R. (2010). Behavioral activation for depression: A clinician's guide. New York: Guilford.

McCauley, E., Gudmundsen, G., Schloredt, K., Martell, C., Rhew, I., Hubley, S., \& Dimidjian, S. (2015). The adolescent behavioral activation program: Adapting behavioral activation as a treatment for depression in adolescence. Journal of Clinical Child \& Adolescent Psychology, 1-14. doi: 10.1080/15374416.2014.979933.

Miller, S. D., \& Duncan, B. L. (2000) The outcome rating scale. Chicago: Author.

National Institute for Health and Care Excellence (2015). CG28 Depression in children and young people: Identification and management in primary, community and secondary care. NICE clinical guideline 28, issued September 2005. Updated March 2015. Retrieved from http://www.nice.org.uk/guidance/cg28

Orchard, F., Pass, L., Marshall, T. \& Reynolds, S. (2016). Clinical characteristics of adolescents referred for treatment of depressive disorders. Child and Adolescent Mental Health. doi: 10.1111/camh.12178. 
Pass, L., Brisco, G., Hodgson, E., \& Reynolds, S. (2015). Brief Behavioural Activation for depressed adolescents (Brief BA) therapist guide. Unpublished manual, University of Reading.

Pass, L., \& Reynolds, S. (2014). Treatment manual for Brief Behavioural Activation (Brief BA) for depressed adolescents. Unpublished manual, University of Reading.

Pass, L., Brisco, G., \& Reynolds, S. (2015). Adapting brief Behavioural Activation (BA) for adolescent depression: A case example. The Cognitive Behaviour Therapist, 8, e17. doi: $10.1017 / \mathrm{S} 1754470 \mathrm{X} 15000446$

Pass, L., Whitney, H., \& Reynolds, S. (2016). Brief Behavioural Activation (BA) for adolescent depression: Working with complexity and risk. doi: $10.1177 / 1534650116645402$.

Polanczyk, G. V., Salum, G. A., Sugaya, L. S., Caye, A., \& Rohde, L. A. (2015). Annual research review: A meta-analysis of the worldwide prevalence of mental disorders in children and adolescents. Journal of Child Psychology and Psychiatry, 56, 345-365. doi: 10.1111/jcpp.12381.

Richards, D. A., Ekers, D., McMillan, D., Taylor, R. S., Byford, S., Warren, F. C., ... Finning, K. (2016). Cost and Outcome of Behavioural Activation versus Cognitive Behavioural Therapy for Depression (COBRA): a randomised, controlled, non-inferiority trial. The Lancet. Advance online publication. doi:10.1016/S0140-6736(16)31140-0.

Ruggiero, K. J., Morris, T. L., Hopko, D. R., \& Lejuez, C. W. (2005). Application of Behavioral Activation Treatment for Depression to an adolescent with a history of child maltreatment. Clinical Case Studies, 2, 1-17. doi: 10.1177/1534650105275986.

Skinner, B. (1953). Science and human behavior. New York: Free Press. 
Stasiak, K., Parkin, A., Seymour, F., Lambie, I., Crengle, S., Pasene-Mizziebo, E., \& Merry, S. (2013). Measuring outcome in child and adolescent mental health services: Consumers' views of measures. Clinical Child Psychology and Psychiatry, 18, 519-535. doi: $10.1177 / 1359104512460860$.

Thirlwall, K., Cooper, P. J., Karalus, J., Voysey, M., Willetts, L., \& Creswell, C. (2013). Treatment of child anxiety disorders via guided parent-delivered cognitive-behavioural therapy: randomised controlled trial. The British Journal of Psychiatry, 203, 436444. doi: 10.1192/bjp.bp.113.126698.

Verboom, C. E., Sijtsema, J. J., Verhulst, F. C., Penninx, B. W., \& Ormel, J. (2014). Longitudinal associations between depressive problems, academic performance, and social functioning in adolescent boys and girls. Developmental Psychology, 50, 247. doi: 10.1037/a0032547.

Vivyan, C. (2014). ACE log (http://www.getselfhelp.co.uk/freedownloads2.htm).

Weisz, J. R., McCarty, C. A., \& Valeri, S. M. (2006). Effects of psychotherapy for depression in children and adolescents: a meta-analysis. Psychological Bulletin, 132, 132-149. doi: 10.1037/0033-2909.132.1.132.

\section{Disclosure statement}

None of the authors of this manuscript have any current or potential conflicts of interest. 
Table 1: Key adaptations in Brief BA from BATD-R with examples

\section{Key adaptation in Brief BA Example in Brief BA \\ from BATD-R}

Consideration of

developmental and cognitive

constraints in adolescent

depression
- Session by session workbooks

- Use of diagrams/images

- Adolescent case examples illustrating each Brief

BA concept

- Simplified life areas and values overview

- Collaborative agenda setting each session

- Framing of activity monitoring as a 'getting to know you' tool

- Emphasis on personal values that might not align with family/peers/teachers review

- Parent session by session workbook mirroring adolescent content

- Collection of parent questionnaires

Considering values that are relevant to young people, and
- $\quad$ Specific discussion and exploration of young person's values 
how to collaboratively $\quad-\quad$ Clinician guide to investigating values with young investigate these people

Working with potential $\quad-$ Inclusion of problem-solving technique

conflict between young

- Contracts with parents in session

person's values and those of

others (e.g. parents, friends)

- Initial discussion about potential conflicts in values

Embedded use of Routine

- Questionnaires collected at every session

Outcome Monitoring (ROMs)

- Explicit question on risk at each session

including risk assessment

- Questionnaires included in clinician session

checklist and used in every supervision session 
Table 2: Comparison of BATD-R and Brief BA session content

Session Adult BATD-R session content

Adolescent Brief BA session content

number

\begin{tabular}{ll}
\hline 1 Client only & $\begin{array}{l}\text { Young person + Parent for part of } \\
\text { session plus handout and ROMs }\end{array}$ \\
1. Discussion of depression & 1. Brief discussion of adolescent \\
2. Introduction of BATD rationale & depression (focus on adolescent
\end{tabular}

3. Introduction to daily monitoring

presentation including irritability)

(Enjoyment and Importance ratings)

2. Introduction of Brief BA rationale

4. Emphasis on attending all sessions

and completing homework assignments

3. Explore individual BA maintenance

formulation

Homework: Daily monitoring form

4. Introduction to activity monitoring

(Achievement, Closeness, Enjoyment,

and Importance ratings), complete

example together.

Homework: Activity log; Read handout

$2 \quad$ Client only

Young person only (Parent: handout

1. Review of daily monitoring form,

and $R O M s$ )

troubleshooting if necessary

1. Review of activity log (ACE-I

2. Review of BATD rationale ratings), troubleshooting if necessary 
3. Introduction to life areas, values, and 2. Review of Brief BA rationale activities inventory (split across 5

3. Introduction to concept of values

broad domains: Relationships;

Education/ career; Recreation/ Homework: Activity log; Read handout interests; Mind/ body/ spirituality;

Daily responsibilities)

Homework: Daily monitoring form;

Review and edit life areas, values, and activities inventory

$3 \quad$ Client only

1. Review of daily monitoring form

2. Review of life areas, values, and activities Inventory: Review assignment

\section{Activity Selection and Ranking}

Homework: Daily monitoring;

Continue to review and edit life areas, values, and activities inventory;

Review and edit activity selection and ranking
Young person only (Parent: handout

and $R O M s$ )

1. Review of activity log

2. Exploration of young person's values (across 3 broad domains: Me; Things that matter; People that matter).

3. Plan 1 target valued activity

Homework: Complete target activity; Activity log (if possible); Read handout 
$4 \quad$ Client only

1. Review of daily monitoring form.

2. Daily monitoring with planning

Homework: Daily monitoring with activity planning for upcoming week
Young person only (Parent: handout

and $R O M s$ )

1. Review of target activity homework

2. Review of values

3. Plan valued activities across

different life areas and high on ACE-I.

3. Plan 3 target valued activities

Homework: Complete 3 target

activities; Activity log (if possible);

Read handout

Young person only (Parent: handout

and $R O M s$ )

1. Review of daily monitoring with

1. Review of 3 target activities

activity planning form

2. Introduction to Contracts

2. Review of values \& valued activities

3. Daily monitoring with activity

planning for the upcoming week

Homework: Daily monitoring with

activity planning for the upcoming

week; Continue adding/editing

contracts
3. Identify activities currently doing, want to do, and need help to do

4. Plan further valued activities

Homework: Complete planned valued

activities; Activity log (if possible);

Read handout 


\begin{tabular}{ll}
\hline Client only & Young person + Parent for part of \\
1. Review of daily monitoring with & session plus handout and ROMs \\
activity planning & 1. Review of progress (including \\
2. Review Contracts & planned valued activities) \\
3. Daily monitoring with activity & 2. Introduction to Contracts (if \\
planning for the upcoming week & appropriate). \\
Homework: Daily monitoring with & 3. Introduction to problem-solving \\
activity planning for the upcoming & Homework: Complete valued \\
week; Continue adding/editing & activities, use contracts/problem- \\
contracts & solving if needed; Activity log (if \\
\end{tabular}

$7 \quad$ Client only

1. Review daily monitoring with activity planning

2. Life areas, values, and activities inventory: Concept review and edit

3. Daily monitoring with activity planning for the upcoming week
Young person only (Parent: handout

and $R O M s$ )

1. Review of progress: What has

changed? What do I still want to work on?

2. Here and now BA cycle

Homework: Complete valued activities; Activity log (if possible);

Read handout 


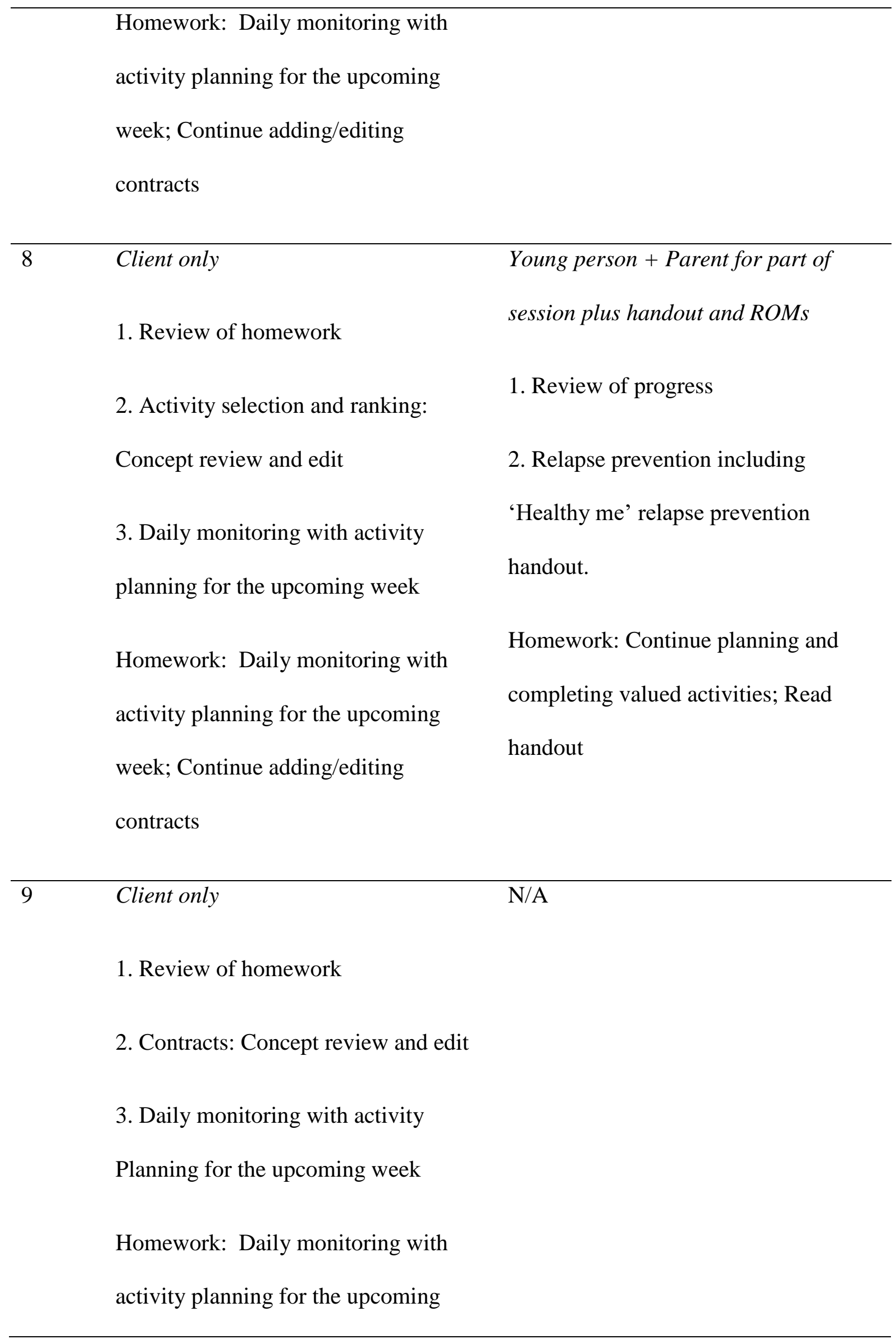


week; Continue adding/editing

contracts

10 and Client only N/A

beyond

1. Review of homework

2. Daily Monitoring With Activity

Planning for the Upcoming Week

3. Preparing for the End of Treatment

Homework: Daily monitoring with

activity planning for the upcoming

week; Continue adding/editing

contracts

Review N/A

Young person + Parent for part of

session and ROMs

1. Review of progress

2. Plan for further input/discharge 
Table 3. Emma's Life Areas and Values

\begin{tabular}{lll}
\hline Life Area & Life Area Example & Value \\
\hline Hobbies & Art and writing \\
Pe & Dosical Health & Getting grades up in Maths \& \\
& & English; Getting a good grade in \\
& & Art \\
\hline The things that matter & Everyday stuff & Getting out the house
\end{tabular}




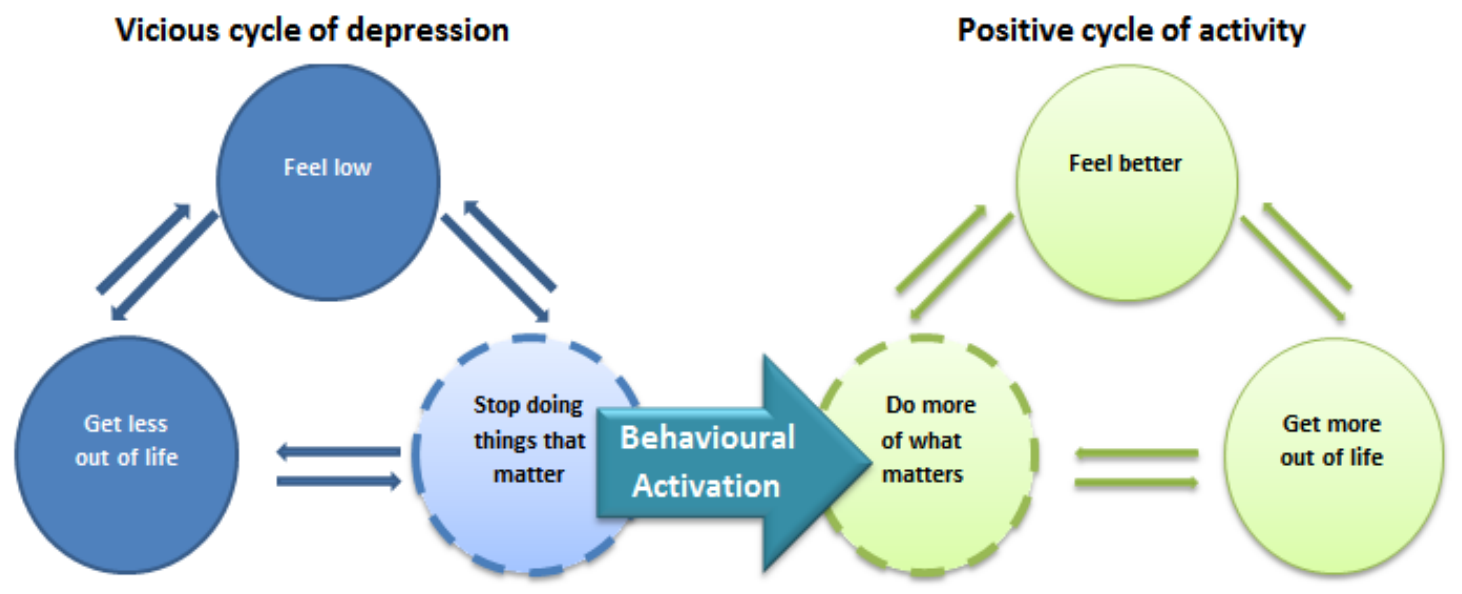

Figure 1. Brief BA model

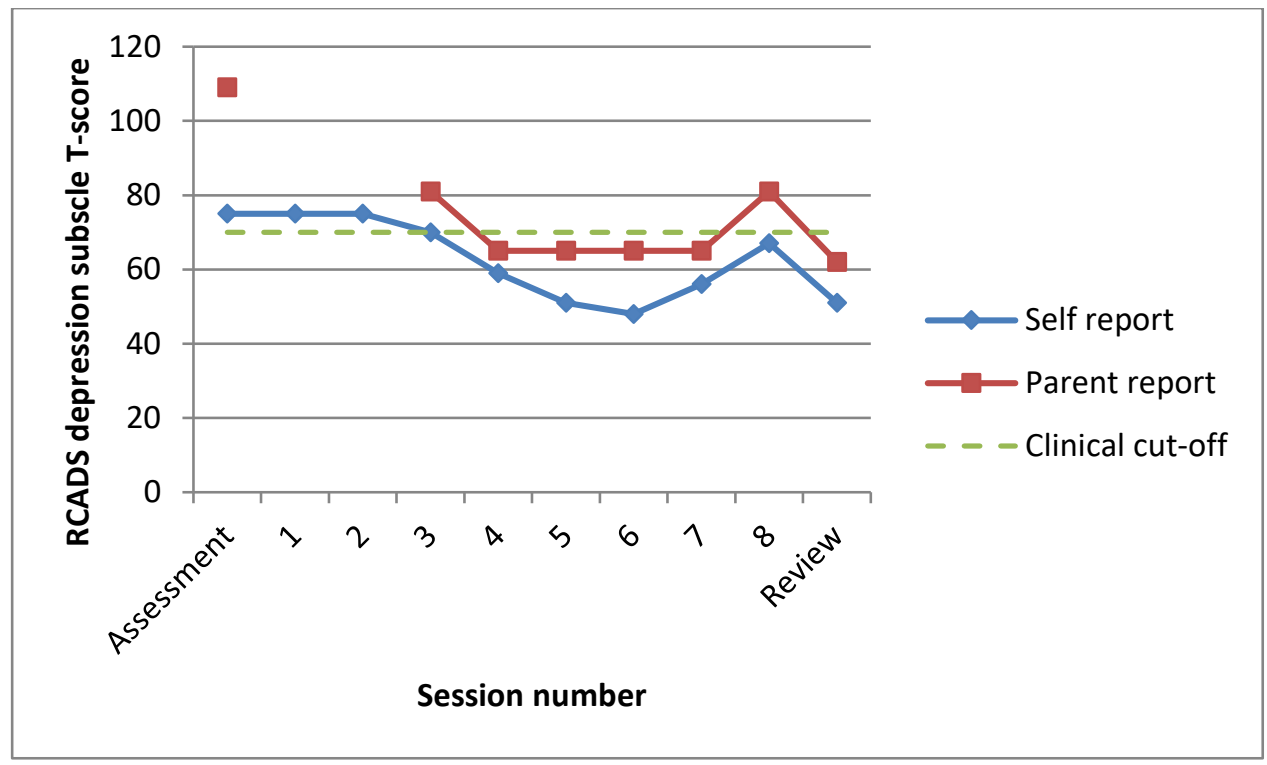

Figure 2. RCADS depression subscale self-report and parent report 


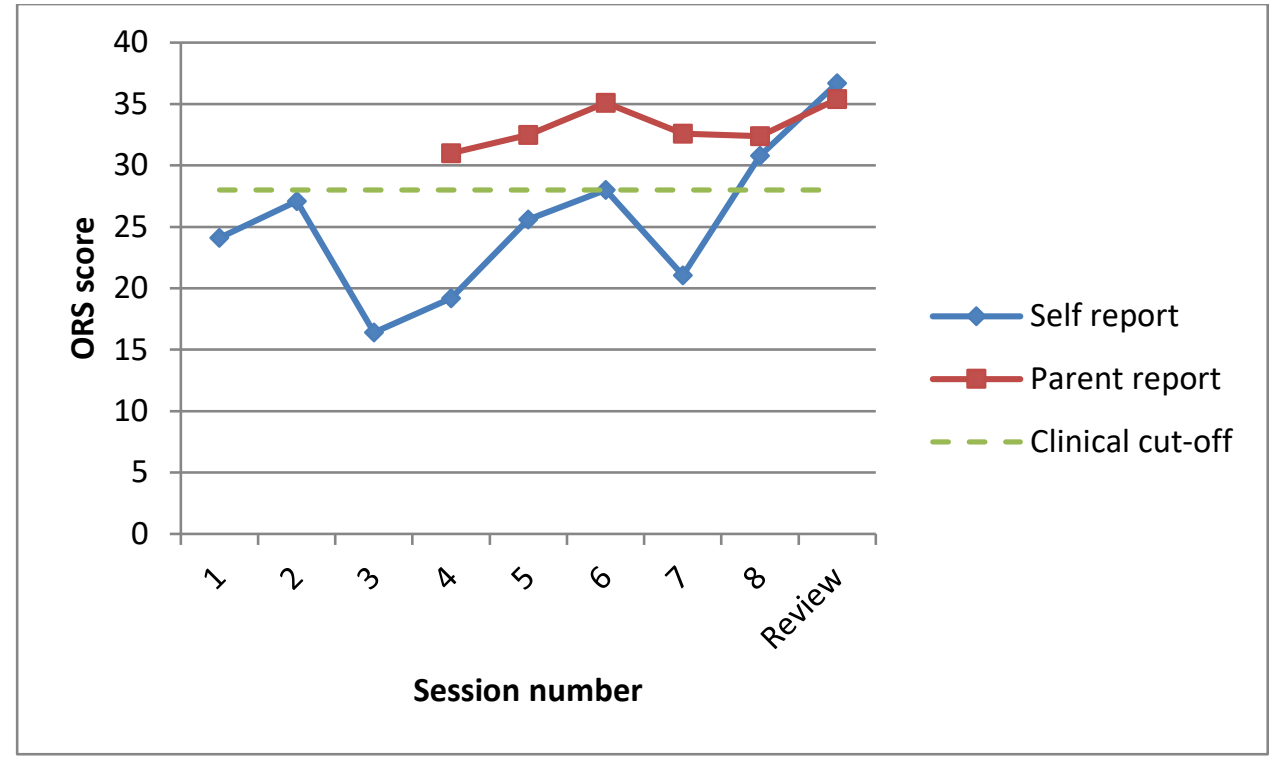

Figure 3. Outcome Rating Scale (ORS) self-report and parent report 